\title{
Packing Graphs: \\ The packing problem solved
}

\author{
Yair Caro * \\ and \\ Raphael Yuster ${ }^{\dagger}$ \\ Department of Mathematics \\ University of Haifa-ORANIM, Tivon 36006, Israel. \\ AMS Subject Classification: 05B05,05B40 (primary), \\ 05B30,51E05,94C30,62K05,62K10 (secondary).
}

Submitted: November 28, 1996; Accepted: December 2, 1996

Dedicated to the memory of Paul Erdős

\begin{abstract}
For every fixed graph $H$, we determine the $H$-packing number of $K_{n}$, for all $n>n_{0}(H)$. We prove that if $h$ is the number of edges of $H$, and $\operatorname{gcd}(H)=d$ is the greatest common divisor of the degrees of $H$, then there exists $n_{0}=n_{0}(H)$, such that for all $n>n_{0}$,

$$
P\left(H, K_{n}\right)=\left\lfloor\frac{d n}{2 h}\left\lfloor\frac{n-1}{d}\right\rfloor\right\rfloor,
$$

unless $n=1 \bmod d$ and $n(n-1) / d=b \bmod (2 h / d)$ where $1 \leq b \leq d$, in which case

$$
P\left(H, K_{n}\right)=\left\lfloor\frac{d n}{2 h}\left\lfloor\frac{n-1}{d}\right\rfloor\right\rfloor-1 .
$$
\end{abstract}

Our main tool in proving this result is the deep decomposition result of Gustavsson.

\section{Introduction}

All graphs considered here are finite, undirected and simple. For the standard graph-theoretic terminology the reader is referred to $[\underline{\mathbf{B o}}]$. Let $H$ be a graph without isolated vertices. An $H$ packing of a graph $G$ is a set $L=\left\{G_{1}, \ldots, G_{s}\right\}$ of edge-disjoint subgraphs of $G$, where each subgraph

*e-mail: zeac603@uvm.haifa.ac.il

†e-mail: raphy@math.tau.ac.il 
THE ELECTRONiC JOURNAL OF COMBINATORICS 4 (1997), \#R1

is isomorphic to $H$. The $H$-packing number of $G$, denoted by $P(H, G)$, is the maximum cardinality of an $H$-packing of $G$. An $H$-covering of a graph $G$ is a set $L=\left\{G_{1}, \ldots, G_{s}\right\}$ of subgraphs of $G$, where each subgraph is isomorphic to $H$, where every edge of $G$ appears in at least one member of $L$. The $H$-covering number of $G$, denoted by $C(H, G)$, is the minimum cardinality of an $H$-covering of $G$. $G$ has an $H$-decomposition if it has an $H$-packing which is also an $H$-covering. The $H$-packing and $H$-covering problems are, in general, NP-Complete as shown by Dor and Tarsi [DoTa]. In case $G=K_{n}$, the $H$-covering and $H$-packing problems have attracted much attention in the last forty years, and numerous papers were written on these subjects (cf. [Br95,Ha,MiMu,CoDi,StKaMu] for various surveys). Special cases of these problems gained particular interest.

1. $P\left(K_{k}, K_{n}\right)$ which has been linked to the various Johnson-Schonheim bounds in Coding Theory [BiEt,BrShSlSm,Sc,Jo]. It is known that $P\left(K_{k}, K_{n}\right)$ is the maximum size of the binary codes of word-length $n$, constant weight $k$, and distance $2 k-2$ or $2 k-3$. Despite of much effort only the cases $k=3$ [ and $k=4$ [2] Sc and $k=4$ [2]] are solved. The case $k=5$ is still open $[\underline{\mathrm{MuYi}}]$.

2. $P\left(C_{k}, K_{n}\right)$ which is the cycle-system packing problem, solved completely only for $k=3, k=4$ [and $k=5[17]$ ScBi and $k=5[\mathbf{1 7}]]$.

3. The packing-covering conjecture for trees saying that $P\left(T, K_{n}\right)=\left\lfloor\left(\begin{array}{l}n \\ 2\end{array}\right) / h\right\rfloor$ and $C\left(T, K_{n}\right)=$ $\left\lceil\left(\begin{array}{l}n \\ 2\end{array}\right) / h\right\rceil$ ( $h$ is the number of edges of $T$ ) provided $n$ is sufficiently large. This conjecture has been proved for all trees on at most 7 vertices $[\underline{\mathbf{R o 8 3}, \mathbf{R o 9 3}}]$.

A central result concerning $H$-decompositions of $K_{n}$ is the theorem of Wilson [Wi] stating that for sufficiently large $n, K_{n}$ has an $H$-decomposition if and only if $e(H) \mid\left(\begin{array}{l}n \\ 2\end{array}\right)$ and $\operatorname{gcd}(H) \mid n-1$ where $\operatorname{gcd}(H)$ is the greatest common divisor of the degrees of $H$. Clearly, if the conditions in Wilson's Theorem hold, then the packing and covering numbers are known.

In this paper we solve all of the conjectures above, for large $n$, as special consequences of a much more general result. In fact, for every $H$, we determine $P\left(H, K_{n}\right)$, for all $n \geq n_{0}(H)$.

Theorem 1.1 Let $H$ be a graph with $h$ edges, and let $\operatorname{gcd}(H)=d$. Then there exists $n_{0}=n_{0}(H)$, such that for all $n>n_{0}$,

$$
P\left(H, K_{n}\right)=\left\lfloor\frac{d n}{2 h}\left\lfloor\frac{n-1}{d}\right\rfloor\right\rfloor,
$$

unless $n=1 \bmod d$ and $n(n-1) / d=b \bmod (2 h / d)$ where $1 \leq b \leq d$, in which case

$$
P\left(H, K_{n}\right)=\left\lfloor\frac{d n}{2 h}\left\lfloor\frac{n-1}{d}\right\rfloor\right\rfloor-1 .
$$


THE ElECtronic Journal of COMBinatorics 4 (1997), \#R1

\section{Proof of the main result}

As mentioned in the abstract, our main tool is the following result of Gustavsson $[\mathbf{G u}]$ :

Lemma 2.1 (Gustavsson's Theorem [ $\underline{\mathbf{G u}}$ )] Let $H$ be a graph with $h$ edges. There exists $N=$ $N(H)$, and $\epsilon=\epsilon(H)>0$, such that for all $n>N$, If $G$ is a graph on $n$ vertices and $m$ edges, with $\delta(G) \geq n(1-\epsilon), \operatorname{gcd}(H) \mid \operatorname{gcd}(G)$, and $h \mid m$, then $G$ has an $H$-decomposition.

It is worth mentioning that $N(H)$ in Gustavsson's Theorem is a rather huge constant; in fact, it is a highly exponential function of $h$.

A sequence of $n$ positive integers $d_{1} \geq d_{2} \geq \ldots \geq d_{n}$ is called graphic if there exists an $n$-vertex graph whose degree sequence is $\left\{d_{1}, \ldots, d_{n}\right\}$. We shall need the following theorem of Erdős and Gallai $[\mathbf{E r G a}]$, which gives a necessary and sufficient condition for a sequence to be graphic.

Lemma 2.2 (Erdös and Gallai [ErGa)] The sequence $d_{1} \geq d_{2} \geq \ldots \geq d_{n}$ of positive integers is graphic if and only if its sum is even and for every $t=1, \ldots, n$

$$
\sum_{i=1}^{t} d_{i} \leq t(t-1)+\sum_{i=t+1}^{n} \min \left\{t, d_{i}\right\} .
$$

Proof of Theorem 1.1: Given $H$, we choose $n_{0}=n_{0}(H)=\max \left\{N(H), \frac{2 h}{\epsilon(H)}, 8 h\right\}$, where $N(H)$ and $\epsilon(H)$ are as in Lemma 2.1. Now let $n>n_{0}$. Let $n-1=a \bmod d$, where $0 \leq a \leq d-1$. Let $n(n-1-a) / d=b \bmod (2 h / d)$, where $0 \leq b \leq 2 h / d-1$. Note that since $d=\operatorname{gcd}(H)$ and $2 h$ is the sum of the degrees of $H, 2 h / d$ must be an integer. Also note that $(n-1-a) / d$ is an integer, and so $b$ is well-defined. We shall use the obvious fact that $h \geq d(d+1) / 2$, since $\delta(H) \geq d$. This means that

$$
n>n_{0} \geq 8 h>4 d^{2}>(a+d)^{2} .
$$

Another useful fact is that $b d+n a$ is even since if $d$ is even then $a$ and $n$ have different parity, and if $d$ is odd then $2 h / d$ is even and so if $b$ is odd then $a$ and $n$ are both odd, and if $b$ is even then either $n$ is even or $a$ is even. In the first part of the proof we shall give a lower bound for $P\left(H, K_{n}\right)$, and in the second part we shall give an upper bound for $P\left(H, K_{n}\right)$, and notice that the lower and upper bounds coincide.

Proving a lower bound for $P\left(H, K_{n}\right)$ : We shall first assume that $a \neq 0$. Our first goal is to show the existence of an $n$-vertex graph which has $b$ vertices with degree $d+a$, and $n-b$ vertices with degree $a$. For this purpose we shall use Lemma 2.2, with $d_{i}=a+d$ for $i=1, \ldots, b$ and $d_{i}=a$ 
THE ELECTRONIC JOURNAL OF COMBINATORICS 4 (1997), \#R1

for $i=b+1, \ldots, n$. Notice first that the sum of the sequence is $b d+n a$ and this number is even as mentioned above. Let $1 \leq t \leq a+d$. In this case, (1) holds since

$$
\sum_{i=1}^{t} d_{i} \leq t(a+d)=t(t-1)+t(a+d-t+1) \leq t(t-1)+(a+d)(a+d-1)=
$$

$t(t-1)+(a+d)^{2}-(a+d)<t(t-1)+n-(a+d) \leq t(t-1)+(n-t) \leq t(t-1)+\sum_{i=t+1}^{n} \min \left\{t, d_{i}\right\}$.

For $a+d \leq t \leq n$ we shall prove that (1) holds by induction on $t$, where the base case $t=a+d$ was proved above. If $t>a+d$ we use the induction hypothesis to derive that

$$
\begin{gathered}
\sum_{i=1}^{t} d_{i}=d_{t}+\sum_{i=1}^{t-1} d_{i} \leq d_{t}+(t-1)(t-2)+\sum_{i=t}^{n} \min \left\{t, d_{i}\right\}= \\
d_{t}+\min \left\{t, d_{t}\right\}-2(t-1)+t(t-1)+\sum_{i=t+1}^{n} \min \left\{t, d_{i}\right\} \\
\leq(a+d)+(a+d)-2(a+d)+t(t-1)+\sum_{i=t+1}^{n} \min \left\{t, d_{i}\right\}=t(t-1)+\sum_{i=t+1}^{n} \min \left\{t, d_{i}\right\} .
\end{gathered}
$$

Thus, there exists a graph $G^{*}$ having $b$ vertices with degree $d+a$ and $n-b$ vertices with degree $a$. Consider $G=K_{n} \backslash G^{*}$. Clearly, $d \mid \operatorname{gcd}(G)$, and $G$ has $m$ edges where

$$
\left.m=\left(\begin{array}{l}
n \\
2
\end{array}\right)-\frac{b d+n a}{2}=\frac{d}{2}\left(\frac{n(n-1-a)}{d}-b\right)\right)=0 \bmod h .
$$

Also note that $\delta(G) \geq n-1-a-d=n\left(1-\frac{1+a+d}{n}\right) \geq n(1-\epsilon(H))$, since $n>n_{0} \geq \frac{2 h}{\epsilon(H)}$. Thus, $G$ satisfies the conditions of Lemma 2.1, and therefore $G$ has an $H$-decomposition. This means that

$$
\left.P\left(H, K_{n}\right) \geq P(H, G)=\frac{m}{h}=\frac{d}{2 h}\left(\frac{n(n-1-a)}{d}-b\right)\right)=\left\lfloor\frac{d n}{2 h}\left\lfloor\frac{n-1}{d}\right\rfloor\right\rfloor .
$$

We now deal with the case $a=0$. If $b=0$ then $K_{n}$ has an $H$-decomposition according to Wilson's Theorem $[\underline{\mathbf{W i}}]$, (or according to Lemma 2.1), so, trivially,

$$
P\left(H, K_{n}\right)=\frac{\left(\begin{array}{l}
n \\
2
\end{array}\right)}{h}=\frac{d n}{2 h} \frac{n-1}{d}=\left\lfloor\frac{d n}{2 h}\left\lfloor\frac{n-1}{d}\right\rfloor\right\rfloor .
$$

If $b>d$ we may delete from $K_{n}$ a subgraph $G^{*}$ on $b$ vertices which is $d$ regular (this is doable since $b d+n a=b d$ is even). As in the case where $a \neq 0$, the remaining graph $G=K_{n} \backslash G^{*}$ satisfies the conditions of Lemma 2.1 and therefore

$$
P\left(H, K_{n}\right) \geq P(H, G)=\frac{\left(\begin{array}{c}
n \\
2
\end{array}\right)-\frac{b d}{2}}{h}=\left\lfloor\frac{\left(\begin{array}{c}
n \\
2
\end{array}\right)}{h}\right\rfloor=\left\lfloor\frac{d n}{2 h} \frac{n-1}{d}\right\rfloor=\left\lfloor\frac{d n}{2 h}\left\lfloor\frac{n-1}{d}\right\rfloor\right\rfloor .
$$


THE ELECTRONIC JOURNAL OF COMBINATORICS 4 (1997), \#R1

Finally, if $1 \leq b \leq d$ then we can delete from $K_{n}$ a subgraph $G^{*}$ on $b+\frac{2 h}{d}$ vertices which is $d$ regular. Note that this can be done since $h \geq d(d+1) / 2$ which implies $d \leq \frac{2 h}{d}<\frac{2 h}{d}+b$. Also, if $d$ is odd then $b$ and $\frac{2 h}{d}$ are both even, so $b+\frac{2 h}{d}$ is even. Once again, the remaining graph $G=K_{n} \backslash G^{*}$ satisfies the conditions of Lemma 2.1 and we get

$$
P\left(H, K_{n}\right) \geq P(H, G)=\frac{\left(\begin{array}{c}
n \\
2
\end{array}\right)-\frac{(b+(2 h / d)) d}{2}}{h}=\frac{\left(\begin{array}{c}
n \\
2
\end{array}\right)-\frac{b d}{2}}{h}-1=\left\lfloor\frac{\left(\begin{array}{c}
n \\
2
\end{array}\right)}{h}\right\rfloor-1=\left\lfloor\frac{d n}{2 h}\left\lfloor\frac{n-1}{d}\right\rfloor\right\rfloor-1 .
$$

Proving an upper bound for $P\left(H, K_{n}\right)$ : Let $L$ be an arbitrary $H$-packing of $K_{n}$. Let $s$ denote the cardinality of $L$. Let $G$ denote the edge-union of all the members of $L$. $G$ contains $s h$ edges. Thus $G^{*}=K_{n} \backslash G$ contains $\left(\begin{array}{l}n \\ 2\end{array}\right)-s h$ edges. The degree of every vertex in $G$ is $0 \bmod d$ and so the degree of every vertex in $G^{*}$ is $a \bmod d$. Therefore, the number of edges in $G^{*}$ satisfies

$$
\left(\begin{array}{l}
n \\
2
\end{array}\right)-s h=\frac{a n+c d}{2}
$$

for some non-negative integer $c$. In particular, $\left(\begin{array}{l}n \\ 2\end{array}\right)=\frac{a n+c d}{2} \bmod h$. This, in turn, implies that $n(n-1-a) / d=c \bmod (2 h / d)$. Thus, we must have $c \geq b$. Therefore,

$$
s=\frac{\left(\begin{array}{l}
n \\
2
\end{array}\right)-\frac{a n+c d}{2}}{h} \leq \frac{\left(\begin{array}{l}
n \\
2
\end{array}\right)-\frac{a n+b d}{2}}{h}=\left\lfloor\frac{d n}{2 h}\left\lfloor\frac{n-1}{d}\right\rfloor\right\rfloor .
$$

Since $L$ was an arbitrary $H$-packing, we have

$$
P\left(H, K_{n}\right) \leq\left\lfloor\frac{d n}{2 h}\left\lfloor\frac{n-1}{d}\right\rfloor\right\rfloor .
$$

The only remaining case is $a=0$ and $1 \leq b \leq d$. In this case, we cannot have $c=b$. This is because every non-isolated vertex of $G^{*}$ has degree at least $d$, and therefore there are at least $d(d+1) / 2$ edges in $G^{*}$, i.e $c d / 2 \geq d(d+1) / 2$, which implies $c \geq d+1$, but $b \leq d$. We must, therefore have $c \geq b+2 h / d$. Therefore,

$$
s=\frac{\left(\begin{array}{l}
n \\
2
\end{array}\right)-\frac{a n+c d}{2}}{h} \leq \frac{\left(\begin{array}{l}
n \\
2
\end{array}\right)-\frac{a n+(b+2 h / d) d}{2}}{h}=\left\lfloor\frac{d n}{2 h}\left\lfloor\frac{n-1}{d}\right\rfloor\right\rfloor-1 .
$$

\section{Concluding remarks}

1. Theorem 1.1, applied to $H=K_{k}$ yields, for $n \geq n_{0}(k)$, that

$$
P\left(K_{k}, K_{n}\right)=\left\lfloor\frac{n}{k}\left\lfloor\frac{n-1}{k-1}\right\rfloor\right\rfloor,
$$

unless $k-1 \mid n-1$ and $n(n-1) /(k-1) \bmod k$ is less than $k$ and greater than 0 , in which case the above formula should be reduced by 1 . This solves, in particular, the related problem in Coding Theory mentioned in the introduction. 
THE ELECTRONIC JOURNAL OF COMBINATORICS 4 (1997), \#R1

2. Theorem 1.1, applied to $H=C_{k}$ yields, for $n \geq n_{0}(k)$, that

$$
P\left(C_{k}, K_{n}\right)=\left\lfloor\frac{n}{k}\left\lfloor\frac{n-1}{2}\right\rfloor\right\rfloor
$$

unless $n$ is odd and $\left(\begin{array}{l}n \\ 2\end{array}\right)=1,2 \bmod k$.

3. If $n \geq n_{0}(H)$ and $\operatorname{gcd}(H)=1$, then $P\left(H, K_{n}\right)=\left\lfloor\frac{\left(\begin{array}{c}n \\ 2\end{array}\right)}{e(H)}\right\rfloor$. Note that by first deleting from $K_{n}$ any set of $b<e(H)$ edges where $b=\left(\begin{array}{c}n \\ 2\end{array}\right) \bmod e(H)$, the remaining graph satisfies the conditions in Gustavsson's Theorem, and since the set of deleted edges may be chosen as a subgraph of $H$ we have $C\left(H, K_{n}\right)=\left\lceil\frac{\left(\begin{array}{l}n \\ 2\end{array}\right)}{e(H)}\right\rceil$, solving, in particular, the packing-covering conjecture for trees.

Our approach allows us to solve the covering problem as well. This is done in a forthcoming paper $[\underline{\mathbf{C a Y u}}]$.

\section{Acknowledgment}

The authors wish to thank N. Alon, T. Etzion, R. Mullin, J. Schonheim and Y. Roditty for useful discussions, helpful information, and sending important references.

\section{References}

[1] S. Bitan and T. Etzion, The last packing number of quadruples and cyclic SQS, Design, Codes and Cryptography 3 (1993), 283-313.

[2] A.E. Brouwer, Optimal packing of $K_{4}$ 's into a $K_{n}$, J. Combin. Theory, Ser. A 26 (1979), 278-297.

[3] A.E. Brouwer, Block Designs, in: Chapter 14 in "Handbook of Combinatorics", R. Graham, M. Grötschel and L. Lovász Eds. Elsevier, 1995.

[4] A. Brouwer, J. Shearer, N. Sloane and W. Smith, A new table of constant weight codes, IEEE Trans. Inform. Theory 36 (1990), 1334-1380.

[5] B. Bollobás, Extremal Graph Theory, Academic Press, 1978.

[6] Y. Caro and R. Yuster, Covering graphs: The covering problem solved, submitted.

[7] C.J. Colbourn and J.H. Dinitz, CRC Handbook of Combinatorial Design, CRC press 1996. 
THE ELECTRONiC JOURNAL OF COMBINATORICS 4 (1997), \#R1

[8] D. Dor and M. Tarsi, Graph decomposition is NPC - A complete proof of Holyer's conjecture, Proc. 20th ACM STOC, ACM Press (1992), 252-263.

[9] P. Erdös and T. Gallai, Graphs with prescribed degrees of vertices (Hungarian), Math. Lapok 11 (1960), 264-274.

[10] T. Gustavsson, Decompositions of large graphs and digraphs with high minimum degree, Doctoral Dissertation, Dept. of Mathematics, Univ. of Stockholm, 1991.

[11] H. Hanani, Balanced incomplete block designs and related designs, Discrete Math. 11 (1975), 255-369.

[12] S.M. Johnson, A new upper bound for error-correcting codes, IEEE Trans. Inform. Theory 8 (1962), 203-207.

[13] W.H. Mills and R.C. Mullin, Coverings and packings, in: Contemporary Design Theory: A collection of Surveys, 371-399, edited by J. H. Dinitz and D. R. Stinson. Wiley, 1992.

[14] R.C. Mullin and J. Yin, On packing of pairs by quintuples $v=3,9,17(\bmod 20)$, Ars Combinatoria 35 (1993), 161-171.

[15] Y. Roditty, Packing and covering of the complete graph with a graph $G$ of four vertices or less, J. Combin. Theory, Ser. A 34 (1983), 231-243.

[16] Y. Roditty, Packing and covering of the complete graph IV, the trees of order 7, Ars Combinatoria 35 (1993), 33-64.

[17] A. Rosa and S. Znam, Packing pentagons into complete graphs: how clumsy can you get, Discrete Math. 128 (1994), 305-316

[18] J. Schonheim, On maximal systems of k-tuples, Studia Sci. Math. Hungar. (1966), 363-368.

[19] J. Schonheim and A. Bialostocki, Packing and covering of the complete graph with 4-cycles, Canadian Math. Bull. 18 (1975), 703-708.

[20] R.G. Stanton, J.G. Kalbfleisch and R.C. Mullin, Covering and packing designs, Proc. $2^{\text {nd }}$ Chapel Hill Conf. on Combinatorial Mathematics and its applications. Univ. North Carolina, Chapel Hill (1970) 428-450.

[21] R. M. Wilson, Decomposition of complete graphs into subgraphs isomorphic to a given graph, Congressus Numerantium XV (1975), 647-659. 